\title{
Potential of CMS as a high-energy neutrino scattering experiment
}

\author{
P. Foldenauer®, ${ }^{1, *}$ F. Kling $\odot^{2,3, \dagger}$ and P. Reimitz $\odot^{4, *}$ \\ ${ }^{1}$ Institute for Particle Physics Phenomenology, Durham University, Durham DH1 3LE, United Kingdom \\ ${ }^{2}$ Theory Group, SLAC National Accelerator Laboratory, Menlo Park, California 94025, USA \\ ${ }^{3}$ Deutsches Elektronen-Synchrotron DESY, Notkestrasse 85, 22607 Hamburg, Germany \\ ${ }^{4}$ Instituto de Física, Universidade de Sāo Paulo, 05508-090 Sāo Paulo, Brazil
}

(Received 26 August 2021; accepted 23 November 2021; published 21 December 2021)

\begin{abstract}
With its enormous number of produced neutrinos the LHC is a prime facility to study the behavior of high-energy neutrinos. In this paper we propose a novel search strategy for identifying neutrino scattering via displaced appearing jets in the high granularity calorimeter of the CMS endcap in the high luminosity run of the LHC. We demonstrate in a cut and count based analysis how the enormous hadronic background can be reduced while keeping most of the neutrino signal. This paper serves as a proof of principle study to illustrate the feasibility of the first direct observation of high-energy neutrinos coming from $W$ decays.
\end{abstract}

DOI: 10.1103/PhysRevD.104.113005

\section{INTRODUCTION}

The matter content of Standard Model (SM) consists of three generations of quarks and leptons. Of these the three neutral leptons, i.e., the neutrinos, are arguably the most elusive. Being the lightest particles of the SM, not only are their masses and the mechanism responsible for generating them still undetermined, but they are also very difficult to probe experimentally since they only participate in weak interaction. Nevertheless, neutrinos have been detected from a vast variety of sources, including nuclear reactors, beam dumps, the atmosphere, the Earth, the Sun, supernovae, and even extragalactic astrophysical sources.

In this study, we consider a novel complementary neutrino source; the Large Hadron Collider (LHC). As the highest-energy collider experiment, the LHC is also the source of the most energetic human made neutrinos. However, the chance that those neutrinos scatter in any of the detector components is very low. To illustrate this, we can estimate the probability $P$ of a neutrino with energy $E_{\nu}$ to interact in a meter of material with density $\rho$ and obtain $P \sim 4 \times 10^{-13} \times\left[E_{\nu} / \mathrm{GeV}\right] \times\left[\rho /\left(\mathrm{g} \mathrm{cm}^{-3}\right)\right]$. Therefore, neutrinos at the LHC have only been observed indirectly as a missing transverse momentum. Nevertheless, as proposed already decades ago [1-9], it is also possible in principle to directly observe LHC neutrinos through their

\footnotetext{
*patrick.foldenauer@durham.ac.uk felixk@slac.stanford.edu *peter@if.usp.br
}

Published by the American Physical Society under the terms of the Creative Commons Attribution 4.0 International license. Further distribution of this work must maintain attribution to the author(s) and the published article's title, journal citation, and DOI. Funded by SCOAP. scattering, despite the small interaction rate. Indeed, this idea was reconsidered in recent years. As a result, two new experiments with the goal to measure LHC neutrinos will start their operation during Run 3 of the LHC; FASER $\nu$ [10,11] and SND@LHC [12,13].

Neutrinos at the LHC can originate from the weak decay of a variety of SM particles of different masses, ranging from pions to electroweak gauge bosons. This is illustrated in Fig. 1, where we show the distribution of neutrinos, weighted by their interaction probability $P$, in terms of their rapidity and energy. We can see that the population of neutrinos cluster around $p_{T} \sim m / 2$, where $m$ is the mass of their parent particle, as denoted by the dotted lines. Therefore, depending on the detector location and probed energy range, different production modes can dominate the neutrino flux.

Both FASER $\nu$ and SND@LHC are emulsion based detectors located in the far-forward direction, corresponding to large rapidities, about $480 \mathrm{~m}$ downstream from the ATLAS interaction point. At this location, they can be placed on or close to the beam collision axis, where the rate of neutrino interactions per detector volume is maximized. Both experiments use an emulsion based technology. In the case of FASER $\nu$, the emulsion detector is followed by a magnetized spectrometer, while for SND@LHC the emulsion is interleaved with tracking layers and followed by a muon system. The feasibility of neutrino detection using emulsion detectors at this location was recently demonstrated by the FASER Collaboration, which reported the observation of the first neutrino interaction candidates at the LHC [15]. Both experiments will mainly detect neutrinos from pion, kaon, and charm decays, and their corresponding neutrino fluxes have recently been discussed in Ref. [16].

In Fig. 1, we also include an ensemble of neutrinos from $W$-boson decay which populate a more central rapidity 


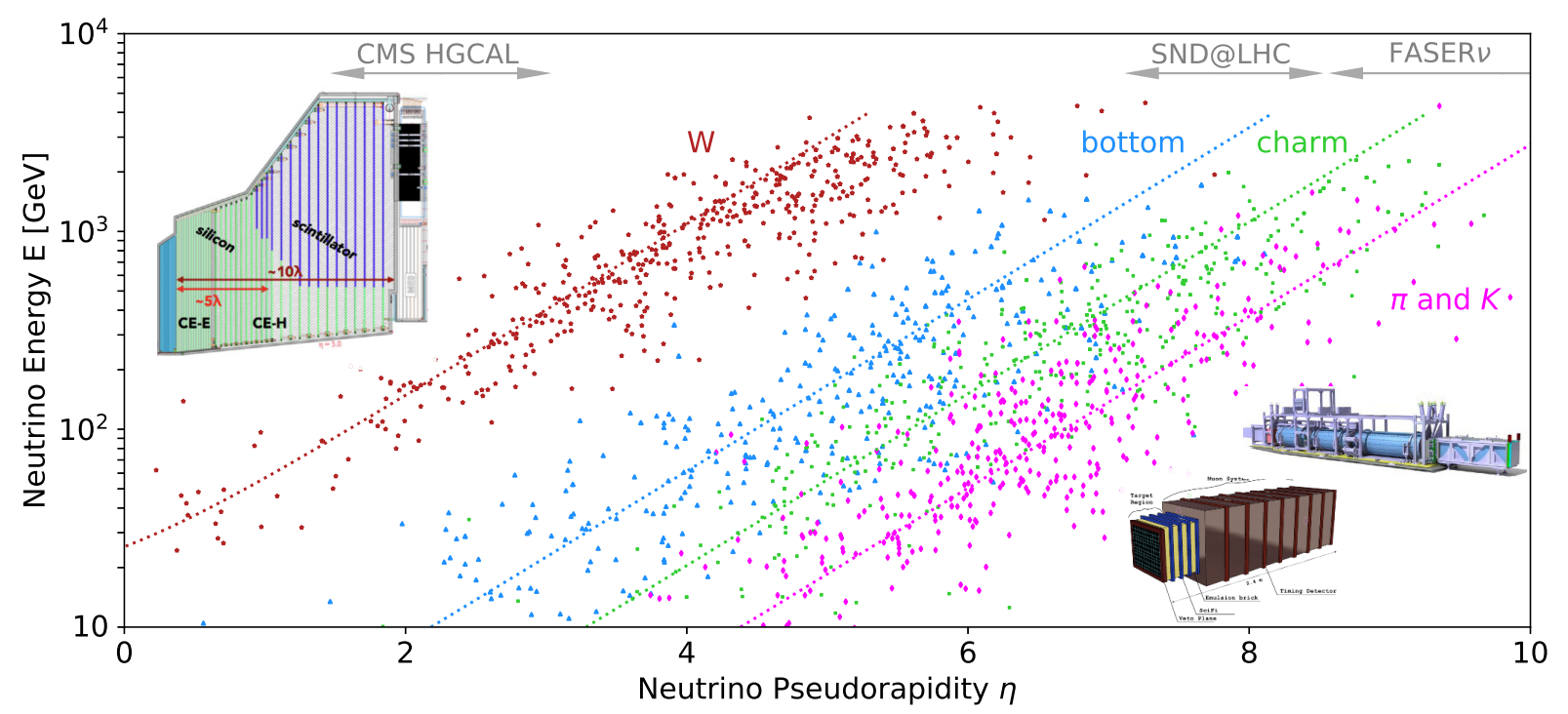

FIG. 1. Neutrino Distribution. The scattered points illustrate the distribution of neutrinos, as simulated using Pythia 8 and weighted by the interaction probability, in terms of the neutrino pseudorapidity $\eta$ and the neutrino energy $E$. The differently colored populations denote neutrinos from the decay of $W$-bosons (red), bottom hadrons (blue), charmed hadrons (green), and light hadrons (magenta), where the number of points is the same for all production modes. The metastable pion and kaons are required to decay before colliding with the beam pipe or being deflected by the LHC magnets. The dashed lines correspond to the typical transverse momentum $p_{T} \sim m / 2$ where $m$ is the mass of the parent particle. We also show the pseudorapidity coverage and illustrations of the CMS HGCAL [14], and the dedicated forward LHC neutrino detectors SND@LHC [12,13] and FASER $\nu[10,11]$.

range. These neutrinos can scatter in the calorimeters of the LHCs large multipurpose detectors, ATLAS or CMS, and leave observable energy deposits. The main challenge is to distinguish those neutrino scattering events from the scattering of other long-lived neutral particles, such as neutrons or neutral kaons, whose production and interaction rates are many orders of magnitude larger.

In this study, we present an analysis strategy to separate the neutrino signal from hadronic backgrounds, which demonstrates that a search for neutrino interactions in the main LHC detectors can be performed in principle. We will focus on a particularly promising neutrino target; the high granularity calorimeter (HGCAL) upgrade of the CMS endcap [14]. As we will see, it provides both a fine spatial segmentation and pileup rejection capabilities, which will play a crucial role in suppressing the hadronic backgrounds.

The rest of the paper is organized as follows: In Sec. II we give a brief description of the future CMS HGCAL and its unique spatial and temporal resolution. In Sec. III we lay out the search strategy and discuss the relevant analysislevel cuts necessary for detecting neutrino scattering in the HGCAL. Finally, in Sec. IV we summarize our results and give a brief outlook on the future potential of this type of search strategy.

\section{THE CMS HIGH GRANULARITY CALORIMETER}

In this section, we want to briefly summarise the main characteristics of the future CMS HGCAL.

\section{A. Detector geometry and design}

During the high-luminosity run of LHC (HL-LHC) one of the main challenges for LHC detectors are backgrounds from the very high number of collisions occurring in the same bunch crossing - the pileup. In order to discriminate interesting events from the background, LHC detectors will therefore need excellent spatial and temporal resolution. This requirement, together with the fact that the expected fluence of neutrons and the level of ionizing radiation is significantly higher than in current runs (in particular those close to the beam pipe) has led to a novel design of the CMS endcap calorimeter for the HL-LHC [14,17].

The planned upgrade of the CMS endcap calorimeterthe HGCAL-is a sampling detector located at $|z|=3.2 \mathrm{~m}$ from the interaction point (IP) and extending to $|z|=5.2 \mathrm{~m}$. It covers the pseudorapidity range $1.5<|\eta|<3.0$ and mostly utilizes silicon as the active material. One of the main arguments for silicon is its good performance under the high expected radiation levels with neutron fluences of up to $10^{16} \mathrm{n}_{\text {eq }} / \mathrm{cm}^{2}$ and doses of $2 \mathrm{MGy}$ expected for the HL-LHC. The HGCAL consists in total of three different segments [14]. The first segment closest to the IP is the electromagnetic calorimeter (ECAL) consisting of a silicon-tungsten detector with 28 sampling layers totaling $26 X_{0}$ radiation lengths and $1.7 \lambda$ hadronic interaction lengths. The individual silicon cells have a size of $0.5 \mathrm{~cm}^{2}-1 \mathrm{~cm}^{2}$ in the transverse plane. This finely granulated detector design allows a high-resolution measurement of the lateral development of electromagnetic showers. This helps to achieve a good two-shower separation 
and the observation of narrow jets. For example, for photon showers of $p_{T}>40 \mathrm{GeV}$ a resolution of better than $4 \mathrm{mrad}$ will be achievable [14]. Following the electromagnetic calorimeter comes a 24 layer hadronic sampling calorimeter (HCAL) of $\sim 8.5 \lambda$ length, which employs two different detector designs. The first HCAL segment is a siliconstainless steel sampling detector that has the same transverse cell size as the ECAL. The second segment of the HCAL consists of a plastic scintillator-stainless steel sampling detector with a transverse cell size of $4 \mathrm{~cm}^{2}-30 \mathrm{~cm}^{2}$. In total, the HGCAL has a hadronic interaction length of $\sim 10 \lambda$, such that most of the hadronic shower is contained in the detector, and due to the finely granulated segmentation can be observed with high resolution, both in the transversal and longitudinal direction.

Since we are interested in the measurement of neutrino scattering, the main issue will be the mitigation of neutral hadronic backgrounds. Due to its excellent spatial and temporal resolution, the CMS HGCAL is the best candidate for this task at the LHC. In particular the excellent timing capabilities will be crucial in reducing the amount of neutral background from pileup as we will discuss in a moment. For comparison, the ATLAS liquid argon barrel and endcap calorimeters [18] have a coarser spatial segmentation leading to a worse angular resolution. Furthermore, these calorimeters themselves do not provide any timing information, which makes suppression of neutral hadronic backgrounds from pileup extremely challenging. The CMS barrel calorimeter does provide timing information, but only in the ECAL part of the detector [19]. Furthermore, the spatial granularity of the detector is inferior to the HGCAL. Both ATLAS [20] and CMS [21] are planning to install separate timing detectors in their Upgrade-II before the HL-LHC run. However, this will only help to mitigate pileup from charged tracks and will not help reduce backgrounds from neutral hadrons. Similarly, the LHCb Collaboration has plans for incorporating a dedicated timing detector in front of the ECAL [22], which again only allows for timing of tracks. Furthermore, $\mathrm{LHCb}$ will collect significantly less luminosity than ATLAS or CMS, making the observation of neutrinos unfeasible due to the small interaction rates.

\section{B. Pileup mitigation with timing}

When analyzing neutrino scattering events in the calorimeter during the high-luminosity run of the LHC, care has to be taken to eliminate potential pileup contamination.

In this context, the CMS endcap HGCAL will be of special importance, since it will be able to mitigate pileup significantly due to its timing capabilities [14]. (For example, neutral $K_{L}^{0}$ with $p_{T}>5 \mathrm{GeV}$ are reconstructed with an efficiency of $>90 \%$ and a resolution of $\lesssim 30 \mathrm{ps}$.)

The typical timing window chosen to remove pileup is $90 \mathrm{ps}$ [14] and corresponds to a path length difference traveled by relativistic particles of $\Delta_{l} \sim 2.7 \mathrm{~cm}$. In order to further enhance the effect of this timing window on pileup mitigation, a novel collision technique for the HL-LHC has been proposed; the so-called crab kissing scheme [23]. In the crab-kissing scheme, the bunches are colliding at a crossing angle. However, before the collision they pass an radio-frequency cavity giving the tail and head of the bunches a kick so that they rotate and collide with maximum spatial overlap [24]. This way the distance over which the various pileup collisions occur is stretched out in space and can be described by a super-Gaussian distribution of order four [25]. The pileup density at the HL-LHC in the optimized case (crab kissing) has a typical spatial extension (full width at half maximum) of $31.4 \mathrm{~cm}$ with the nominal number of expected pileup events being about 130 per bunch crossing [25].

If we approximate the super-Gaussian distribution in the crab-kissing scenario by a uniform distribution, the timing resolution will allow us to reduce the number of pileup events per bunch crossing on average to roughly ${ }^{1}$

$$
N_{\mathrm{pu}} \approx \frac{2.7}{31.4} 130 \sim 11
$$

While in reality, the observed number of pileup events follows a distribution, in this study we will, for simplicity, assume a constant number of pileup events of $N_{\mathrm{pu}}=10$.

\section{NEUTRINO SCATTERING AT LHC}

Highly energetic neutrinos are abundantly produced in the primary $p p$ collisions at the LHC. However, their probability to interact with the main detectors is very low, and their presence only manifests itself as a missing transverse momentum. This could change at the HLLHC, where their abundance is sufficiently large such that a sizable number of neutrinos are expected to scatter with nuclei in the denser part of the detector, e.g., the calorimeter. To be more precise, we expect $\mathcal{O}(10)$ muon neutrinos from $W$-boson decays to interact with the hadronic calorimeters of ATLAS and CMS. In this work we explore the potential of observing such neutrino scattering events with LHC multipurpose detectors in a cut and count based search strategy.

\section{A. Signal and background characteristics}

In the following analysis a signal event is defined as a neutrino scattering with the detector material. The scatterings occur mostly via the charged current interaction at

\footnotetext{
${ }^{1}$ For simplicity we have assumed that the path length difference of $\Delta_{l} \sim 2.7 \mathrm{~cm}$ corresponds to the difference of the vertex position in the $z$-direction. However, since the HGCAL is not positioned exactly along the $z$-axis, the vertex separation is obtained by $\cos \theta \Delta_{l}$, where $\theta$ is the angle of emission of the particle. Nevertheless, e.g., in the center of the angular coverage at $\eta \sim 2.3$ we have $\cos \theta \sim 0.98$, which can safely be ignored.
} 
high energies, where the neutrino converts into a charged lepton and the scattered quark appears as a jet. This provides for a characteristic signature to be searched for in neutrino scattering at the LHC, where a charged lepton and a single energetic jet are produced in a single displaced vertex in the calorimeter with no tracks pointing back to the IP.

As seen in Fig. 1, $W$-boson decays are a promising source of highly-energetic neutrinos with $E \gtrsim 10^{2} \mathrm{GeV}$ for interactions within the CMS HGCAL. In the SM we expect approximately equal decay rates into $W \rightarrow e \nu_{e}, \mu \nu_{\mu}$, and $\tau \nu_{\tau}$ final states at high energies. However, due to the excellent muon reconstruction efficiency of CMS we focus on the muonic decay of the $W$-boson as the most promising signature. The signal is hence given by the subsequent process,

$$
\begin{aligned}
& \text { Production : } q q^{\prime} \rightarrow W \rightarrow \mu_{1} \nu_{\mu}, \\
& \text { Scattering }: \nu_{\mu} N \rightarrow \mu_{2}+\text { jet, }
\end{aligned}
$$

with a primary muon $\mu_{1}$ coming from the production process and a secondary muon $\mu_{2}$ in the scattering. To determine the neutrino-interaction rate inside the detector material, we use the deep inelastic scattering neutrino interaction cross sections evaluated at leading order as presented in Ref. [10] (for recent reviews on neutrino cross sections see Refs. [26,27]). Assuming a dominant quark over antiquark contribution in the initial state, the neutrino cross section scales as $d \sigma /(d x d y) \sim 1$ and the antineutrino cross section scales as $d \sigma /(d x d y) \sim\left(E_{\mu_{2}} / E_{\nu}\right)^{2}$. This leads to a larger overall scattering rate of neutrinos compared to antineutrinos.

The dominant backgrounds to this process are isolated muons accompanied by a long-lived neutral hadron interacting with the detector material. Possible candidates to fake the displaced jet of a neutrino signal are neutrons, $K_{L}^{0}$ 's, $\Lambda$ 's, or $\Xi^{0}$. Those neutral hadrons are produced abundantly in hard scattering, the underlying event, or can come from pileup and are very likely to interact with the detector material. The leading backgrounds including an isolated muon along with these hadrons are

(i) The production of a leptonically-decaying $W$ boson in association with additional neutral hadrons

$$
q q^{\prime} \rightarrow W+\mathrm{QCD} \rightarrow \mu_{1}+\mathrm{QCD},
$$

(ii) Heavy-quark production in association with additional neutral hadrons, where a muon is produced in heavy hadron decay

$$
q q^{\prime} \rightarrow b / c+\mathrm{QCD} \rightarrow \mu_{1}+\mathrm{QCD} .
$$

In both cases, the displaced jet is caused by the subsequent scattering of a neutral hadron in the back parts of the calorimeter,

$$
\text { neutral hadron }+N \rightarrow \text { jet. }
$$

In this study, we neglect further subleading sources of muons, such as the decays of $Z$ bosons or in-flight decays of light long-lived hadrons. In the following parton-level based analysis the label $j$ is used for the final state jet-muon system in the scattering process. Likewise, we use the term jet for the total final state particle content. As an example, $E_{j}=E_{\mu_{2}}+E_{\text {had }}$ denotes the energy of the incident particle or, equivalently, the total displaced jet energy, and $E_{\text {had }}$ is the energy carried by hadronic particles produced in the scattering process. In reality, we expect that a typical scattering produces a cone of $\mathcal{O}(10)$ particles that defines the jet [28]. In a more refined analysis including a full detector simulation, we would, then, have to specify a jetfinding algorithm in order to define the jet.

\section{B. Simulation}

Since the goal of this study is to demonstrate the feasibility of detecting neutrino scatterings at the LHC, we perform all simulations for a data set of $3 \mathrm{ab}^{-1}$ at leading order, including parton shower and hadronization. In particular, all signal and background event samples are simulated with PYTHIA8.2 [29,30]. As discussed in Sec. II, we include a constant number of $N_{\mathrm{pu}}=10$ pileup events for every Monte Carlo event. In this study, no full detector simulation was applied. However, we note that the cuts applied in this analysis are chosen conservatively to account for the anticipated detector resolutions. For example, the $R$ separation cuts as used in this paper are chosen large enough to be resolved by the spatial $2 D$ segmentation of the high-resolution CMS HGCAL.

\section{Analysis strategy}

The events of interest consist of one muon and one displaced jet appearing the hadronic calorimeter. All generated samples for signal and background require a muon in the range $|\eta|<4$, according to the planned updated tracker and muon detector at CMS [31-33]. To reduce background from hadronic activity, we discard any displaced jets occurring in the first radiation length of the HCAL. Therefore, we require the displaced jet to appear in the endcap HCAL $(1.5<|\eta|<3)$ at two to ten interaction lengths.

The further analysis strategy is presented in Table I. All cuts in our cut and count analysis are set in a very basic way and leave room for improvement in future searches for high-energy neutrinos. We start with a trigger muon requirement. It is followed by applying a jet isolation cut. Since all signal events are coming from $W$ production, we require the neutrino and muon to reconstruct the $W$ mass. Furthermore, we use the fact that a neutrino scattering process is producing a secondary muon. Finally, we focus 
TABLE I. Cut-flow table with number of neutral hadron background and neutrino signal events for the cut selection $R_{\text {iso }, \mu_{1}}>0.1, R_{\text {iso }, j}>0.1, m_{\text {inv }} \in[66,99], E_{\mu_{2}} / E_{\mathrm{j}}>0.33$, and $E_{\mathrm{j}}>160 \mathrm{GeV}$. See text for details.

\begin{tabular}{lcc}
\hline \hline Cuts & Hadrons & Neutrinos \\
\hline Isolated muon & $1.02 \times 10^{11}$ & 7.59 \\
Isolated jet & $8.63 \times 10^{10}$ & 7.05 \\
$W$ mass & $1.92 \times 10^{9}$ & 6.55 \\
Secondary muon & $3.49 \times 10^{5}$ & 5.48 \\
$E_{\mathrm{j}}>160 \mathrm{GeV}$ & 3.52 & 3.60 \\
\hline \hline
\end{tabular}

on the high-energy tail of the spectrum to enhance the signal to background ratio.

\section{Isolated central muon}

As a first basic cut, we require an isolated central muon with

$$
R_{\mathrm{iso}, \mu_{1}}>0.1, \quad p_{T, \mu_{1}}>20 \mathrm{GeV}, \quad\left|\eta_{\mu_{1}}\right|<2.4 .
$$

Throughout the analysis, we only consider hadrons and charged leptons with $E>10 \mathrm{GeV}$ in the calculation of the separation $R=\sqrt{\Delta \eta^{2}+\Delta \phi^{2}}$ of two particles where $\phi$ is the azimuthal angle about the LHC beam axis. If a certain particle is required to be isolated, its distance $R_{\text {iso }}$ to any hadron or charged lepton has to be greater than a certain specified value. In the hard QCD production of $c \bar{c}$ and $b \bar{b}$ quark pairs, the muons are produced in the decay of the heavy hadron, mostly forward with low $p_{T}$ and

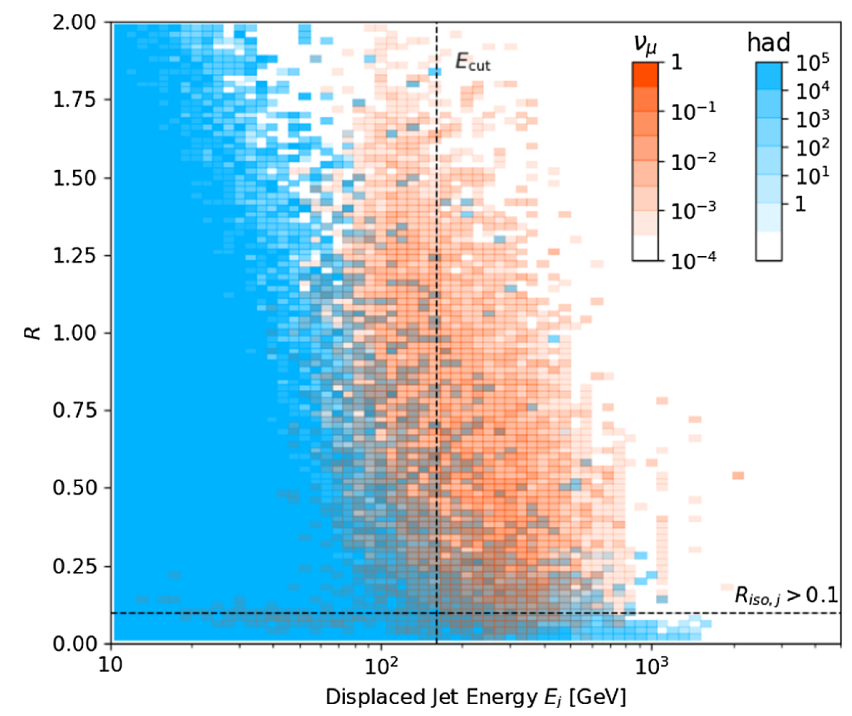

accompanied by another meson from the same decay of $B$ and $D$ mesons. Hence, the muon trigger requirement, $p_{T, \mu_{1}}>20 \mathrm{GeV}$ and $\left|\eta_{\mu_{1}}\right|<2.4$, as well as the isolation are reducing the amount of background events significantly.

\section{Isolated jet}

The signal scattering event can be faked by neutral hadron scatterings in the detector. Here we use that energetic neutral hadrons are mostly produced as part of an energetic, and hence collimated, hadronic shower. To distinguish between neutral hadrons and neutrinos, we therefore apply a jet isolation cut,

$$
R_{\text {iso }, j}>0.1 \text {. }
$$

As can be seen in Table I, this does not have a large effect on the overall number of background events, which is dominated by low-energy events. However, the number of background events in the relevant high-energy bins is reduced significantly as seen in the left panel of Fig. 2 . This figure also indicates that an energy-dependent $R$ cut could be applied as well to improve the signal/background separation even further.

\section{W mass cut}

Additionally, we require the muon and neutrino jet to reconstruct the $W$ mass. Therefore, we set a cut on their invariant mass inspired by the ATLAS $W$-boson search [34],

$$
66 \mathrm{GeV}<m_{\mu \nu}<99 \mathrm{GeV}
$$

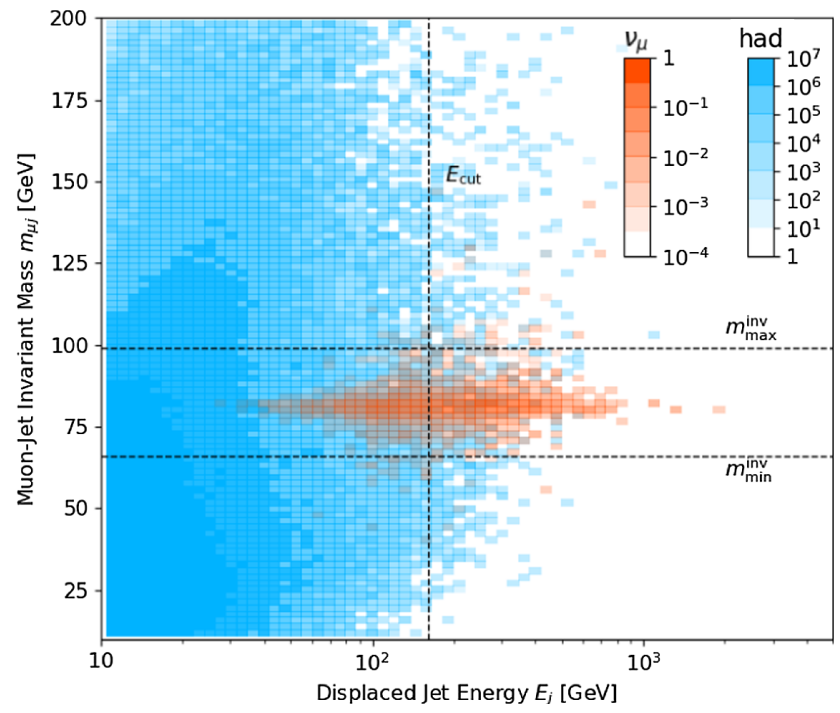

FIG. 2. Left: Distribution of signal (red) and background (blue) events in the jet energy versus the $R$ plane after applying the isolated muon cut. All events below the dashed horizontal line and left of the dashed vertical line will be rejected in the analysis by the jet isolation $R_{\text {iso, } j}>0.1$ and energy cut $E_{\text {cut }}>160 \mathrm{GeV}$, respectively. Right: Signal (red) and background (blue) histograms in the jet energy versus muon-jet invariant mass plane after applying the isolated jet cut. The two dashed horizontal lines indicate the window of selected events after the $W$ mass cut $66 \mathrm{GeV}<m_{\mu \nu}<99 \mathrm{GeV}$. 
in the muon-neutrino system where $\left(p_{\mu_{1}}+p_{\nu}\right)^{2}=m_{\mu \nu}^{2}$. As seen in the right plot of Fig. 2, this encloses the neutrino signal events nicely whereas a large part of the background is removed.

\section{Secondary muon}

Besides different incident scattering particles, another distinguishing feature between the processes in Eq. (2) and Eq. (5) is the presence of a secondary muon produced in the neutrino scattering process. We can use the additional muon as an extra handle to isolate the signal, and search for it with the CMS endcap muon stations located behind the HGCAL covering $1.2<|\eta|<2.4$ [35]. Unlike the trigger muon, the secondary muon is a standalone muon track not matching with any tracker track. We refrain from including efficiency factors and leave this to a detailed detector simulation. Besides, as will be explained below, we will apply an energy condition for the secondary muon which reduces the possibility of misidentification by lowenergy neutral hadrons even further.

Charged current muon-neutrino interactions almost always produce an energetic secondary muon. However, in some rare cases energetic secondary muons can also be produced in the interactions of neutral hadrons with the calorimeter, for example via the in-flight decay of pions produced in these collisions, causing a background for the neutrino interaction signal. To determine the fraction of events, both for the muon-neutrino interaction signal and the neutral hadron interaction background, that contains an energetic secondary muons, we have performed a separate simulation using PYTHIA8 [29]. We have done this by simulating the collisions of these particles with a fixed iron target, using the NCTEQ15 [36] parton distribution function

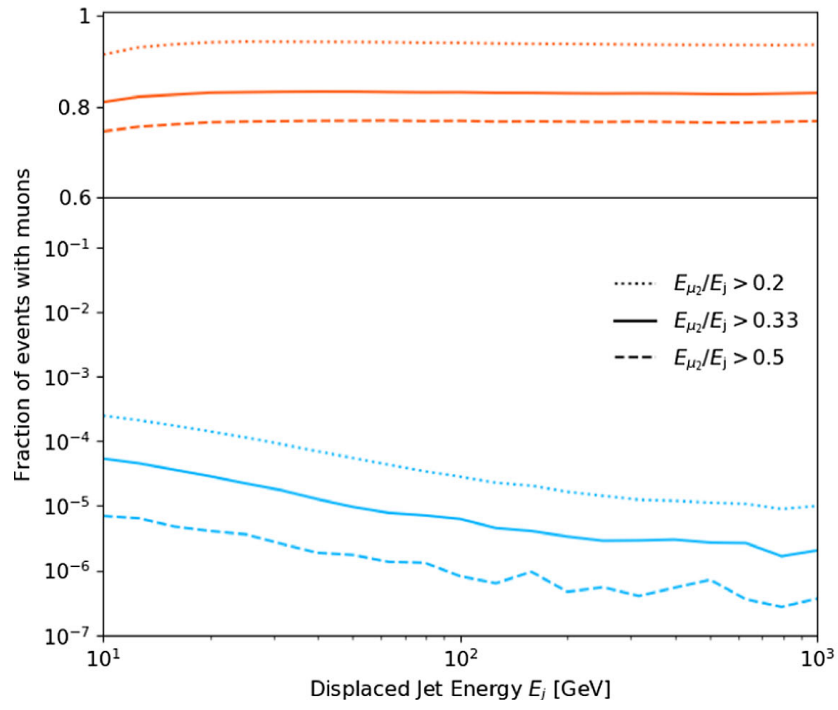

for iron, at different incident particle energies between $10 \mathrm{GeV}$ and $1 \mathrm{TeV}$. Neutral hadrons are represented by neutrons, which contribute the majority of neutral hadrons in this analysis, and about 30 million events are generated for each beam energy. For simplicity, we have assumed that these secondary muons are detected with $100 \%$ efficiency in the CMS muon endcap station. In a more detailed study one would have to perform a fully-fledged detector simulation for the charged current neutrino interactions. Such a study is also necessary to investigate any additional possible backgrounds from fake muons. However, for the purpose of this proof of principle study, we assume that this background is negligible.

For neutral hadron collisions with energies below $E<100 \mathrm{GeV}$, muons are mainly produced in decays of pions and kaons, which we require to decay within one nuclear interaction length, $\lambda_{\text {int }}=18.5 \mathrm{~cm}$. Above $E>100 \mathrm{GeV}$, the secondary muons in background events are dominantly produced in prompt decays; for example, from $\eta, \omega$, and $\phi$ mesons. Charm decays seem to constitute a subdominant component. Contrary to muons of neutrino interactions, most muons produced in the interactions of neutral hadrons with the HGCAL are soft and carry away only a small fraction of the incident particle energy.

The left panel of Fig. 3 shows the fraction of events where muons obtain $20 \%, 33 \%$, or $50 \%$ of the energy of the initial particle. We can see that the fraction of signal events with secondary energetic muons remains well above $70 \%$ for all cut scenarios. We note that antineutrino interactions lead to a larger muon energy fraction compared to neutrino interaction, due to the additional $\left(E_{\mu_{2}} / E_{\nu}\right)^{2}$ factor in the scattering cross section mentioned earlier. In comparison, the amount of events in hadronic processes is suppressed significantly if the produced muon is required to inherit a

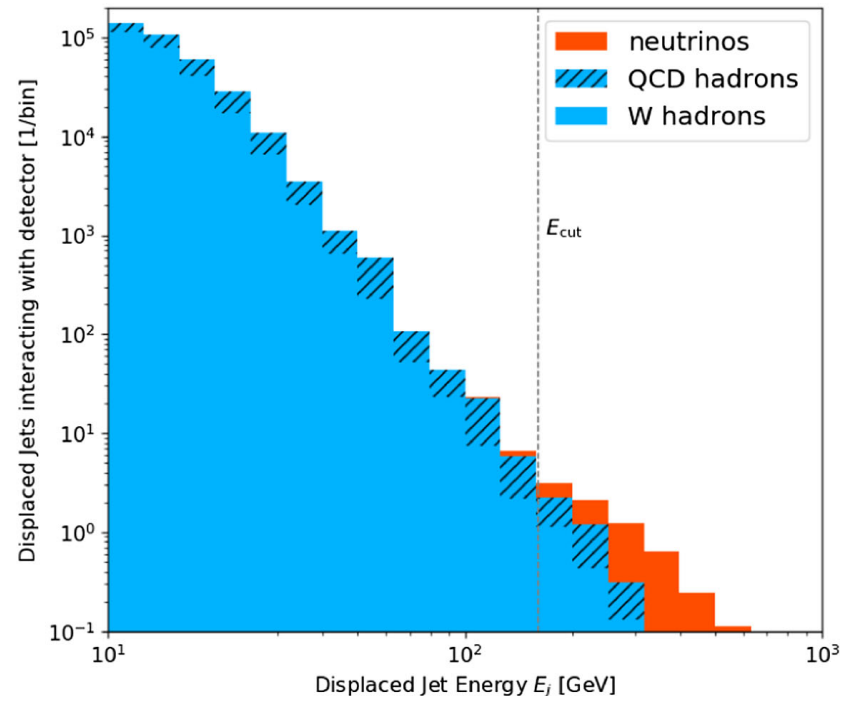

FIG. 3. Left: Fraction of muon-neutrino interaction events (red) and neutral hadron interaction events (blue) containing a muon which carries more than 20\% (dashed), 33\% (solid), and 50\% (dashed) of the energy, as function of the displaced jet energy. Right: Neutrino scattering events (red) and neutral hadron events (blue) after all cuts. The vertical line illustrates the energy cut applied in our analysis. 
substantial fraction of the initial energy. Hence, we require the hardest muon originating from the detector material collision to carry away

$$
E_{\mu_{2}} / E_{\mathrm{j}}>0.33 \text {. }
$$

With a fraction of secondary muons originating from neutral hadron-detector material collisions of roughly $10^{-4}$, this turns out to be a very effective handle to suppress neutral hadron backgrounds.

\section{Energy cut}

Neutrinos coming from $W$ bosons are expected to be more energetic while neutral hadron backgrounds tend to be softer, especially after requiring the displaced jet to be isolated. Hence, we only consider events with displaced jet energies above $E_{\text {cut }}>160 \mathrm{GeV}$ in order to increase the number of signal events over background events.

Starting with about $10^{11}$ background events and $\mathcal{O}(10)$ neutrino events at the muon trigger-level cut, we finally obtain comparable rates after all cuts. While reducing the number of events only mildly in the signal region, we can almost entirely erase the background in the signal region above $E_{\text {cut }}>160 \mathrm{GeV}$. In the right plot of Fig. 3 we present the results after all cuts except for the energy cut. Neutral hadron interactions coming from $c \bar{c}$ and $b \bar{b}$ events are labeled as QCD hadrons. The background smoothly decreases towards higher-displaced jet energies. This makes it easy to subtract the background from the signal events accumulating at the high energy tail of the distribution. To sum up, the basic cut and count analysis presented in this paper shows that there is the potential to see high-energy neutrino scatterings at the LHC. Despite only having a very limited amount of neutrino scattering events inside the CMS HGCAL, the large hadronic background can be very successfully reduced in a way that signal events dominate the high-energy tail of the displaced jet energy spectrum. Already, with very basic cuts as set in this paper, we count approximately an equal number of $\sim 4$ signal and background events above $E_{j}>160 \mathrm{GeV}$.

\section{Sources of systematic uncertainties}

Since the analysis performed in this proof of principle study mostly relies on truth-level Monte Carlo events generated with Pythia, there are a number of potential sources of systematic uncertainties we have to take into account. Focusing on the theoretical description and neglecting any purely experimental effects, we consider the following list of systematic uncertainties:

Primary Interaction: A first class of uncertainties is associated with the simulation of the primary interaction, so the production of $W$ bosons as well as charm and bottom quarks. Here uncertainties arise, for example from the choice of scales or the parton distribution functions, and can affect the production rates and kinematic distributions. However, the $W$-boson [37], charm [38,39], and bottom $[40,41]$ production have been constrained by measurements already, which can be used to estimate and reduce the uncertainties in a data-driven way.

Hadronization: An additional source of uncertainties is associated with the modeling of hadronization. This will have an important impact on the hadron distributions and can affect the effectiveness of the isolation cuts, especially for the isolated jet requirement for neutral hadron backgrounds. One approach would be to use tuning uncertainties [42], as implemented for example in the ATLAS A14 tune [43], to estimate the modeling uncertainties. Alternatively, one could use a data-driven approach and for example directly validate the $R_{\mathrm{iso}, \mu_{1}}$ distribution for muons from $W$ decay which should be the same as the $R_{\mathrm{iso}, j}$ distribution for neutrinos.

Secondary interaction: There are further uncertainties associated with the modeling of the secondary interactions; for example, on the production rates and kinematics of the produced hadrons as well as muons produced in subsequent decays. In particular, this could affect the effectiveness of the secondary muon cut in Eq. (9), which was found to be a powerful handle to suppress the background. While we have used PYTHIA8 to determine the fraction of events with energetic muons, one could use other dedicated hadronic generators such as EPOS [44], SIBYLL [45], QGSJET [46], or DPMJET $[47,48]$ to estimate the uncertainties associated with the modeling of these collisions. These generators have been tuned to relevant fixed target data, for example from NA61 [49], and would ideally be included in a full GEANT4 [50] simulation to accurately model muons from in-flight pion and kaon decays. It might also be possible to constrain the muon rate in a data-driven way, either using fixed target experiments as suggested in Ref. [51] or directly via an analysis of hadronic shower data in the CMS calorimeters.

Finally, a fully data-driven way to verify our background estimates would be to perform a sideband analysis. For example, as can be seen in the right panel of Fig. 2, the muon-jet invariant mass $m_{\mu j}$ distribution outside the signal window $66 \mathrm{GeV}<m_{\mu j}<99 \mathrm{GeV}$ would be ideally suited for estimating the backgrounds and interpolating them to the signal region.

\section{E. Alternative search strategy}

An orthogonal way to search for neutrino scattering events is in heavy flavor production (i.e., $c \bar{c}$ and $b \bar{b}$ events), where the neutrinos are produced in the decay of heavy meson. For example, two such promising candidates are the decays $D^{0} \rightarrow K^{-} \mu^{+} \nu_{\mu}$ and $B^{0} \rightarrow D^{-} \mu^{+} \nu_{\mu}$. Such decays can typically be identified using heavy-flavor tagging techniques which use the presence of a reconstructed secondary vertex due to the comparably long lifetime of 
$B$ and $D$ hadrons. However, one major difference to the search for neutrinos from $W$ production is that the produced muon is not isolated anymore. On the contrary, one expects to find other particles originating from the heavy hadron decay close by. For example, in the case of $D^{0}$ decay mentioned above, the typical angular separation scales as $R \sim m_{D} / p_{T, D} \sim 2 / 40 \sim 0.05$. This is a major drawback when it comes to background suppression since at HL-LHC one expects a lot of pileup events where the produced muon has a charged meson in its vicinity. Another difficulty is that the neutrino produced in the meson decay is much softer compared to the $W$-boson decay. As can be seen in Fig. 1, neutrinos from $W$ bosons mainly produce displaced jet events with $E>100 \mathrm{GeV}$ in the rapidity range accessible at the HGCAL, while those produced in QCD events preferably distribute in the lower displaced jet energy range around $\sim 50 \mathrm{GeV}$. However, in this energy range the background from soft neutral QCD is significantly larger. While in these heavy-flavor processes the overall neutrino production rate is higher than in $W$ production, the enhanced background levels will most probably require a more complex search strategy including e.g., jet-shape variables. We leave a dedicated analysis of these processes for future work.

\section{CONCLUSIONS AND OUTLOOK}

In this paper we have studied the potential of the future CMS HGCAL to detect scattering of muon-neutrinos produced in $W$-boson decays. In this proof of principle study we have demonstrated that with a suitable choice of simple analysis-level cuts, $\mathcal{O}(10)$ muon-neutrino scattering events can be collected during the HL-LHC. Simultaneously, the enormous expected background rates from neutral hadron scattering can be reduced to a similar level by this same set of cuts. This makes the study of neutrino scattering in the $100 \mathrm{GeV}-1 \mathrm{TeV}$ energy range feasible at the HL-LHC; a kinematic range where very few data exists.

From the left panel of Fig. 3 and the cut-flow table shown in Table I, it can be seen that cutting on the energy carried away by the secondary muon produced in the neutrino scattering is vital for reducing the neutral hadron background. Demanding that at least 33\% of the energy of the incoming neutrino to be carried away the muon decreases the amount of background events by a factor of almost $10^{4}$ while it only reduces the signal by $\sim 17 \%$. Finally, the most important cut for background reduction is the jet-energy correlated with the jet isolation cut. This is illustrated in the left panel of Fig. 2. The right panel of Fig. 3 summarizes the final selection of signal and background events after all cuts are applied, where the vertical dashed line illustrates the final jet energy cut on a sufficiently isolated high-energy displaced jet. As can be seen in the last row of Table I, the jet energy cut reduces the number of background events by another factor of $10^{5}$. Thus, after all cuts are applied one expects to detect $\sim 3.6$ muon-neutrino scattering events and $\sim 3.5$ background events from neutral hadron scattering.

This corresponds to a signal-to-background ratio of $S / \sqrt{B} \sim 1.9$ and makes the first direct observation of neutrinos at a high-energy collider multipurpose experiment feasible if further improvements in the analysis are made. While we have already detected neutrinos with $100 \mathrm{GeV}$ energies in dedicated neutrino experiments at Tevatron, and anticipate detecting more at far-foward neutrino experiments at the LHC, those neutrinos are produced in meson decays. The search strategy presented in this paper allows for the first direct observation of neutrinos produced in decays of $W$ bosons.

The search strategy presented in this work can be optimized by using e.g., two-dimensional cuts. For example, in the left panel of Fig. 2 it can be seen that one in principle a better signal to background discrimination could be achieved by using a two-dimensional cut in the jet energy versus the $R$ plane. Ultimately, this type of search could be even further improved by using a fully-fledged multivariate analysis technique. In this context, one could also include more low-level features like shower-shape variables to discriminate between displaced hadronic and neutrino jets. For this purpose it will be necessary to analyze the detector response in a full Geant 4 simulation.

Apart from the aforementioned qualitative improvements of the analysis, we pointed out in Sec. III that an orthogonal direction would be to search for neutrinos in decays of $B$ and $D$ mesons. A further interesting direction is to study the decay $W \rightarrow \nu_{\tau}\left(\tau \rightarrow \nu_{\tau} \mu \nu_{\mu}\right)$, where the prompt decay of the produced tau produces a muon and muon neutrino. If these are energetic enough they could pass the $W$ mass selection criterion and contribute to the muon-neutrino signal. We leave this for future study.

Beyond the scope of SM neutrino interactions the type of search strategy proposed in this work can be applied to a vast class of (light neutral) new physics like neutrino non standard interactions, light mediators or DM. In this context, it has recently been demonstrated that searches for appearing recoil jets have a unique sensitivity to hadronically-coupled light pseudoscalar DM [52]. Another scenario considers the production of dark sector states via muon interactions with the HCAL [53]. Since these types of searches for appearing displaced jets target a complementary set of physics goals than standard LHC searches, its potential should be further studied in the future to optimally exploit the physics output of the LHC program as a whole.

\section{ACKNOWLEDGMENTS}

The authors want to thank Martin Bauer and Tilman Plehn for early collaboration and valuable input on this project, and Jonathan Feng, Max Fieg and Mauro Valli for feedback on the manuscript. Furthermore, the authors are very grateful to Jakob Salfeld-Nebgen for helpful 
discussions on the experimental details of this analysis. P.F. is funded by the UK Science and Technology Facilities Council (STFC) under Grant No. ST/P001246/ 1. The work of F. K. is supported by the U.S. Department of Energy under Grant No. DE-AC02-76SF00515 and by the
Deutsche Forschungsgemeinschaft under Germany's Excellence Strategy-EXC 2121 Quantum Universe390833306. P. R. acknowledges financial support from the Fundação de Amparo à Pesquisa do Estado de São Paulo (FAPESP) under Contract No. 2020/10004-7.
[1] A. De Rujula and R. Ruckl, Neutrino and muon physics in the collider mode of future accelerators, in SSC Workshop: Superconducting Super Collider Fixed Target Physics (1984), pp. 571-596, http://cds.cern.ch/record/1337790.

[2] F. Vannucci, Neutrino physics at LHC/SSC, Technical Report No. LPNHE-93-03, Paris 6. Lab. Phys. Nucl. Théor. Hautes Enérg., Paris, 1993.

[3] A. De Rujula, E. Fernandez, and J. J. Gomez-Cadenas, Neutrino fluxes at future hadron colliders, Nucl. Phys. B405, 80 (1993).

[4] H. Park, The estimation of neutrino fluxes produced by proton-proton collisions at $\sqrt{s}=14 \mathrm{TeV}$ of the LHC, J. High Energy Phys. 10 (2011) 092.

[5] J. L. Feng, I. Galon, F. Kling, and S. Trojanowski, ForwArd search ExpeRiment at the LHC, Phys. Rev. D 97, 035001 (2018).

[6] S. Buontempo, G. M. Dallavalle, G. De Lellis, D. Lazic, and F. L. Navarria, CMS-XSEN: LHC neutrinos at CMS. Experiment feasibility study, arXiv:1804.04413.

[7] N. Beni et al., Physics potential of an experiment using LHC neutrinos, J. Phys. G 46, 115008 (2019).

[8] N. Beni et al. (XSEN Collaboration), XSEN: a $\nu \mathrm{N}$ cross section measurement using high energy neutrinos from $\mathrm{pp}$ collisions at the LHC, arXiv:1910.11340.

[9] N. Beni et al., Further studies on the physics potential of an experiment using LHC neutrinos, J. Phys. G 47, 125004 (2020).

[10] H. Abreu et al. (FASER Collaboration), Detecting and studying high-energy collider neutrinos with FASER at the LHC, Eur. Phys. J. C 80, 61 (2020).

[11] H. Abreu et al. (FASER Collaboration), Technical proposal: FASERnu, arXiv:2001.03073.

[12] C. Ahdida et al. (SHiP Collaboration), SND@LHC, arXiv:2002.08722.

[13] C. Ahdida et al., SND@LHC-Scattering and neutrino detector at the LHC, Technical Report No. CERN-LHCC2021-003; LHCC-P-016, CERN, Geneva, 2021.

[14] CMS Collaboration, The phase-2 upgrade of the CMS endcap calorimeter, Report Nos. CERN-LHCC-2017-023, CMS-TDR-019, 2017.

[15] H. Abreu et al. (FASER Collaboration), First neutrino interaction candidates at the LHC, Phys. Rev. D 104, L091101 (2021).

[16] F. Kling, Forward neutrino fluxes at the LHC, arXiv: 2105.08270.

[17] R. Rusack (CMS Collaboration), High Granularity Calorimeter for the CMS Endcap at HL-LHC, Technical Report No. CMS-CR-2016-444, CERN, Geneva, 2016.
[18] ATLAS Collaboration, ATLAS liquid argon calorimeter phase-II upgrade: Technical design report, Technical Report No. CERN-LHCC-2017-018; ATLAS-TDR-027, CERN, Geneva, 2017.

[19] CMS Collaboration, The phase-2 upgrade of the CMS Barrel calorimeters, Technical Report No. CERN-LHCC2017-011; CMS-TDR-015, CERN, Geneva, 2017. This is the final version, approved by the LHCC.

[20] ATLAS Collaboration, Technical design report: A highgranularity timing detector for the ATLAS phase-II upgrade, Technical Report No. CERN-LHCC-2020-007; ATLASTDR-031, CERN, Geneva, 2020.

[21] CMS Collaboration, A MIP timing detector for the CMS phase-2 upgrade, Technical Report No. CERNLHCC-2019-003; CMS-TDR-020, CERN, Geneva, 2019.

[22] LHCb Collaboration, Expression of interest for a phase-II LHCb upgrade: Opportunities in flavour physics, and beyond, in the HL-LHC era, Technical Report No. CERNLHCC-2017-003, CERN, Geneva, 2017.

[23] S. Fartoukh, Pile up management at the high-luminosity LHC and introduction to the crab-kissing concept, Phys. Rev. ST Accel. Beams 17, 111001 (2014).

[24] S. Verdú-Andrés, S. Belomestnykh, I. Ben-Zvi, R. Calaga, Q. Wu, and B. Xiao, Crab cavities for colliders: Past, present and future, Nucl. Part. Phys. Proc. 273-275, 193 (2016).

[25] L. E. M. Medrano, R. T. Garcia, G. Arduini, and M. Napsuciale, Effective pile-up density as a measure of the experimental data quality for High-Luminosity LHC operational scenarios, Technical Report No. CERN-ACC-20180003, CERN, Geneva, 2018.

[26] J. A. Formaggio and G. P. Zeller, From eV to EeV: Neutrino cross sections across energy scales, Rev. Mod. Phys. 84, 1307 (2012).

[27] K. S. McFarland, Neutrino interactions, in 61st Scottish Universities Summer School in Physics: Neutrinos in Particle Physics, Astrophysics and Cosmology (SUSSP61) (2008) [arXiv:0804.3899].

[28] A. Ismail, R. M. Abraham, and F. Kling, Neutral current neutrino interactions at FASER $\nu$, Phys. Rev. D 103, 056014 (2021).

[29] T. Sjostrand, S. Mrenna, and P.Z. Skands, A brief introduction to PYTHIA8.1, Comput. Phys. Commun. 178, 852 (2008).

[30] T. Sjöstrand, S. Ask, J. R. Christiansen, R. Corke, N. Desai, P. Ilten, S. Mrenna, S. Prestel, C. O. Rasmussen, and P. Z. Skands, An introduction to PYTHIA8.2, Comput. Phys. Commun. 191, 159 (2015). 
[31] M. Cepeda et al., Report from working group 2: Higgs physics at the HL-LHC and HE-LHC, CERN Yellow Rep. Monogr. 7, 221 (2019).

[32] CMS Collaboration, The phase-2 upgrade of the CMS tracker, Technical Report Nos. CERN-LHCC-2017-009, CMS-TDR-014, CERN, Geneva, 2017.

[33] CMS Collaboration, The phase-2 upgrade of the CMS muon detectors, Technical Report Nos. CERN-LHCC-2017-012. CMS-TDR-016, CERN, Geneva, 2017. This is the final version, approved by the LHCC.

[34] M. Aaboud et al. (ATLAS Collaboration), Measurement of the $W$-boson mass in pp collisions at $\sqrt{s}=7 \mathrm{TeV}$ with the ATLAS detector, Eur. Phys. J. C 78, 110 (2018); 78, 898(E) (2018).

[35] A. M. Sirunyan et al. (CMS Collaboration), Performance of the CMS muon detector and muon reconstruction with proton-proton collisions at $\sqrt{s}=13 \mathrm{TeV}$, J. Instrum. 13, P06015 (2018).

[36] K. Kovarik et al., nCTEQ15-Global analysis of nuclear parton distributions with uncertainties in the CTEQ framework, Phys. Rev. D 93, 085037 (2016).

[37] A. M. Sirunyan et al. (CMS Collaboration), Measurements of the $W$ boson rapidity, helicity, double-differential cross sections, and charge asymmetry in $p p$ collisions at $\sqrt{s}=13$ TeV, Phys. Rev. D 102, 092012 (2020).

[38] R. Aaij et al. (LHCb Collaboration), Measurements of prompt charm production cross-sections in $p p$ collisions at $\sqrt{s}=13 \mathrm{TeV}$, J. High Energy Phys. 03 (2016) 159; 09 (2016) 013(E); 05 (2017) 074(E).

[39] A. Tumasyan et al. (CMS Collaboration), Measurement of prompt open-charm production cross sections in protonproton collisions at $\sqrt{s}=13 \mathrm{TeV}$, arXiv:2107.01476.

[40] V. Khachatryan et al. (CMS Collaboration), Measurement of the total and differential inclusive $B^{+}$hadron cross sections in pp collisions at $\sqrt{s}=13 \mathrm{TeV}$, Phys. Lett. B 771, 435 (2017).

[41] R. Aaij et al. (LHCb Collaboration), Measurement of the $B^{ \pm}$ production cross-section in pp collisions at $\sqrt{s}=7$ and 13 TeV, J. High Energy Phys. 12 (2017) 026.
[42] A. Buckley and H. Schulz, Tuning of MC generator MPI models, Adv. Ser. Dir. High Energy Phys. 29, 281 (2018).

[43] ATLAS PyTHIA8 tunes to $7 \mathrm{TeV}$ data.

[44] T. Pierog, I. Karpenko, J. M. Katzy, E. Yatsenko, and K. Werner, EPOS LHC: Test of collective hadronization with data measured at the CERN Large Hadron Collider, Phys. Rev. C 92, 034906 (2015).

[45] F. Riehn, R. Engel, A. Fedynitch, T. K. Gaisser, and T. Stanev, Hadronic interaction model Sibyll 2.3d and extensive air showers, Phys. Rev. D 102, 063002 (2020).

[46] S. Ostapchenko, Monte Carlo treatment of hadronic interactions in enhanced Pomeron scheme: I. QGSJET-II model, Phys. Rev. D 83, 014018 (2011).

[47] S. Roesler, R. Engel, and J. Ranft, The Monte Carlo event generator DPMJET-III, in International Conference on Advanced Monte Carlo for Radiation Physics, Particle Transport Simulation and Applications (MC 2000) (Springer, Berlin, Heidelberg, 2000).

[48] A. Fedynitch, Cascade equations and hadronic interactions at very high energies, Ph.D. thesis, KIT, Karlsruhe, Dept. Phys., 2015.

[49] A. Aduszkiewicz et al. (NA61/SHINE Collaboration), Measurements of $\pi^{ \pm}, \mathrm{K}^{ \pm}, \mathrm{p}$ and $\overline{\mathrm{p}}$ spectra in proton-proton interactions at $20,31,40,80$ and $158 \mathrm{GeV} / c$ with the NA61/SHINE spectrometer at the CERN SPS, Eur. Phys. J. C 77, 671 (2017).

[50] S. Agostinelli et al. (GEANT4 Collaboration), GEANT4-a simulation toolkit, Nucl. Instrum. Methods Phys. Res., Sect. A 506, 250 (2003).

[51] E. Van Herwijnen, H. Dijkstra, A. Korzenev, and P. Mermod (SHiP Collaboration), Calibrating the SHiP muon-flux using NA61/SHINE, https://cds.cern.ch/record/2213605.

[52] M. Bauer, P. Foldenauer, P. Reimitz, and T. Plehn, Light dark matter annihilation and scattering in LHC detectors, SciPost Phys. 10, 030 (2021).

[53] I. Galon, E. Kajamovitz, D. Shih, Y. Soreq, and S. Tarem, Searching for muonic forces with the ATLAS detector, Phys. Rev. D 101, 011701 (2020). 